\title{
Adaptive multivariable relay protection of reconfigurable distribution networks
}

\author{
Mikhail Sharygin ${ }^{1}$,Vladimir Vukolov ${ }^{1}$, Anton Petrov,,* \\ ${ }^{1}$ R. E. Alekseev Nizhnii Novgorod State Technical University, 603950, Minin Str. 24, Nizhnii Novgorod, Russia \\ ${ }^{2}$ JSC NIPOM, advanced development department, 603140, Lenin Ave., 20, Nizhnii Novgorod, Russia
}

\begin{abstract}
Version of relay protection construction of relative selectivity is proposed, which allows increasing its technical perfection and reliability in microgrids with distributed generation sources. This is done by increasing the dimension of the measurement space along arbitrary axes, introducing new methods of recognizing emergency modes, as well as automating the selection of response parameters. Hardware implementation of protection is described. Recommendations for calculating the settings of multidimensional protection and an example of calculation are given. Protection coordination is carried out by using graphical-analytical methods in automatic mode. Automation of the calculation, elimination of the human factor will make it possible to apply the method for adaptation of protection in the conditions of dynamic change of microgrid topology.
\end{abstract}

\section{Introduction}

In electric power industry, micro-network is a medium or low voltage network, which includes sources of power, distributed generation and which is capable of operating both in isolated mode and in parallel with the combined power system.

Application of distributed generation and possibility to dynamically changing topology of the electrical network negatively affects sensitivity of relay protection devices, which control condition of the network $[1,2]$. Solution to this problem can be an adaptive protection system based on digital technologies and modern communications according to IEC 61850 standard. Such protection devices can detect faults both in the external network, ensuring the micro-network's switching to autonomous operation, and faults in the internal network isolating its least possible part to eliminate the fault [3, 4].

Usually, when a relay protection system is developed, distribution network is divided into local zones covering its individual parts. Connection of a new distributed generation source to the network leads to the need to adjust the protection device settings to ensure required selectivity level, speed and sensitivity at autonomous modes and in parallel operation with the external electrical network. Increase in distributed generation volume within the micro-network makes parameterization of its protection system significantly more complex, and the protection system adaptation to existing operating conditions can be carried out both continuously and discretely, by switching pre-defined groups of settings. In any case, correct protection system operation and fine adjustment is possible only under condition of continuous synchronized measurement of various parameters of the micro-network mode in its nodes with following processing of the information received in a specialized device that controls entire network and is able to process large amounts of information. In case of the micro-network fault, such a device identifies the fault type and location and isolates the faulted part from the rest of the network and cuts-off power generation within this part. At that, the remaining parts can continue normal operation.

\section{Proposed concept for protection of micro-networks with distributed generation sources}

Perspective structure of a relay protection system in micro-networks with distributed generation sources involves two-level protection scheme with mutual redundancy. For the first level, it is proposed to use highspeed differential-logic protection [5] using IEC 61850 data protocol to exchange information between distributed intelligent electronic devices (IED), taking into consideration the electrical network topology and operating modes. The second level involves application of multiparameter protection devices based on statistical principles $[6,7,8]$. In case of a failure of the information communication network, the multiparameter protection device ensures operation of the facility similarly to common protection devices with relative selectivity, while having, by virtue of its principle of action, greater sensitivity.

Use of IEC 61850 protocols allows continuous adjusting and adapting the IED to the existing modes of

* Corresponding author: petrov85@inbox.ru 
the micro-network operation using a centralized or decentralized device for automatic calculation of protection triggering parameters.

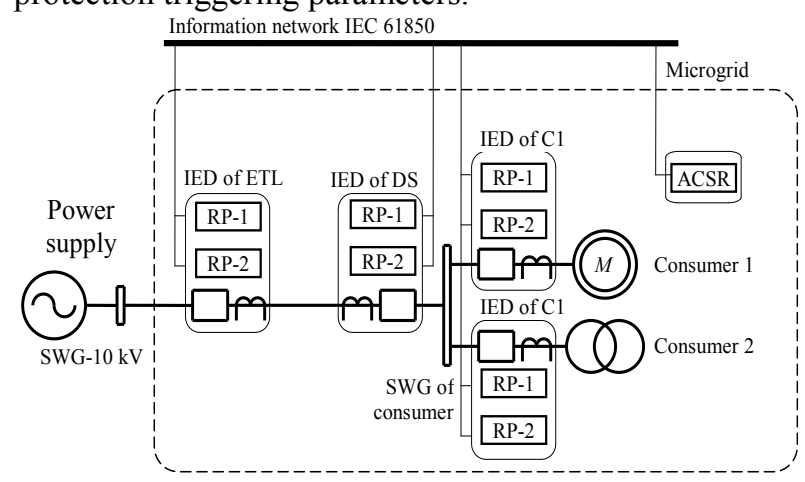

Fig. 1. Application of new protection algorithms for micronetwork: ETL - electrical transmission line; DS - input disconnecting switch; C1, C2 - consumers; RP-1 - first level protection device; RP-2 - second level protection device; SWG - switchgear; ASCR - device for automatic calculation of protection setpoints

The proposed concept allows:

- increasing sensitivity and speed of protection of micronetworks with distributed generation sources;

- increasing stability of the relay protection system to failures of information network, measuring transducers or individual devices;

- ensuring joint operation of the protection devices using different algorithms and operating principles implemented as IED of both centralized and decentralized design.

\section{Perspective principle of emergency mode recognition}

It is reasonable to adapt statistical methods of detection theory as a theoretical basis for development of electric network mode recognition algorithms.

IED, by continuous analysis of parameters of the power network protected element mode, recognizes its current condition as normal (hypothesis $H_{0}$ ) or emergency (hypothesis $H_{1}$ ) operation mode. Criteria values for the facility condition classification are defined so as to minimize average risk $R$ of the protection triggering, in the two possible IED modes they are defined by:

$$
\begin{aligned}
R= & C_{00} P_{0}\left(1-P_{F}\right)+C_{10} P_{0} P_{F}+C_{11} P_{1}\left(1-P_{M}\right)+ \\
& +C_{01} P_{1} P_{M},
\end{aligned}
$$

$C_{00}-$ losses at correct choice of $H_{0}$ hypothesis; $C_{01}-$ losses at incorrect choice of $H_{0}$ hypothesis; $C_{01}-$ losses at incorrect choice of $H_{1}$ hypothesis; $C_{11}-$ losses at correct choice of $H_{1}$ hypothesis; $P_{0}, P_{1}-$ a priori probability of $H_{0}$ и $H_{1}$ hypotheses correctness; $P_{\mathrm{F}}-$ conditional probability of false de-energizing; $P_{\mathrm{M}}-$ conditional probability of undetected alarm mode.

Conditional probability $P_{\mathrm{F}}$ is the probability of event when the protection device attributes current condition of the facility to $H_{1}$ hypothesis while the actual condition matches $H_{0}$ hypothesis. Conditional probability $P_{\mathrm{M}}$, on the contrary, is the probability of attributing by the protection device the facility condition to $H_{0}$ hypothesis while the actual condition matches $H_{1}$ hypothesis:

$$
\begin{aligned}
& P_{F}=P\left(H_{1} \mid H_{0}\right)=\int_{\Psi_{1}} p_{H_{0}}\left(\Phi \mid H_{0}\right) d \Phi ; \\
& P_{M}=P\left(H_{0} \mid H_{1}\right)=\int_{\Psi_{0}} p_{H_{1}}\left(\Phi \mid H_{1}\right) d \Phi,
\end{aligned}
$$

$\Phi$ - vector of monitored mode parameters; $p_{H_{0}}\left(\Phi \mid H_{0}\right)$ - density function of observed parameters under the conditions of the existence of the normal mode $H_{0}$; $p_{H_{1}}\left(\Phi \mid H_{1}\right)$ - density function of observed parameters under the conditions of the existence of the emergency mode $H_{1} ; \Psi_{0}-$ part of the observation space corresponding to permissible condition of the protected facility which does not require to be disconnected from the electrical network; $\Psi_{1}$ - part of the observation space corresponding to non-permissible (emergency) condition of the protected facility.

The density distribution functions of the regime parameters are obtained by conducting a sufficiently large number of statistical experiments on mathematical models of the electric network. For this purpose, in addition to the information necessary for the calculation of traditional relay protections, information on the probabilistic laws of mode distribution is required.

It is obvious that total observation space $\Psi$ is the sum of:

$$
\Psi=\Psi_{0}+\Psi_{1} \text {. }
$$

To increase sensitivity and recognizing ability of the relay protection device, dimension of the monitored space $\Psi$ may be increased, and this will be more effective when using parameters that change maximally in emergency mode $[6,7]$. Thus, different combination of the monitored parameters gives different probabilities of mode recognition.

Features of up-to-date IED make it possible to perform versatile analysis of power network modes by means of universal multidimensional measurementstarting controls that use the most informative monitoring parameters of the network mode.

Bayes criterion according to which the hypothesis is chosen (recognition of the protected facility mode) is [9]:

$$
\begin{cases}H_{0}, & \text { if } \quad \Lambda(\Phi)>\eta \\ H_{1}, & \text { if } \quad \Lambda(\Phi) \leq \eta\end{cases}
$$

where $\eta$ is threshold of likelihood ratio criterion [10]; $\Lambda(\Phi)$ is likelihood ratio:

$$
\Lambda(\Phi)=\frac{p_{H_{1}}\left(\Phi \mid H_{1}\right)}{p_{H_{0}}\left(\Phi \mid H_{0}\right)} .
$$

Offset of the element protection device from all normal modes, in which it must not trigger, gives a value of conditional probability $P_{\mathrm{F}}=0$ which makes it possible to trend the likelihood ratio criterion threshold to infinity: $\eta \rightarrow \infty$ [11].

Recognition of emergency modes of the electric network protected element based on the Bayesian method will allow:

- reducing the risk of erroneous triggering of the relay protection and automatic equipment in the course of decision-making; 
- implementing relay protection and automatic equipment algorithms that take into account actual statistic data of normal and emergency modes of the protected network element;

- developing various new types of automation equipment for both emergency and normal modes.

Let us compare probabilities of short-circuit detection by a supply line protection device at monitoring of various sets of parameters characterizing operating modes of the electric network element which shown is the scheme, in Fig. 2. To do this, based on the results of simulation in different observation spaces $\Psi$, let us draw a curve of probability density function for following characteristic facility modes: normal; short circuit fault in the supply line; short circuit fault in the switchgear of consumer; short circuit fault in the consumer 1 short circuit fault in the consumer 2 .

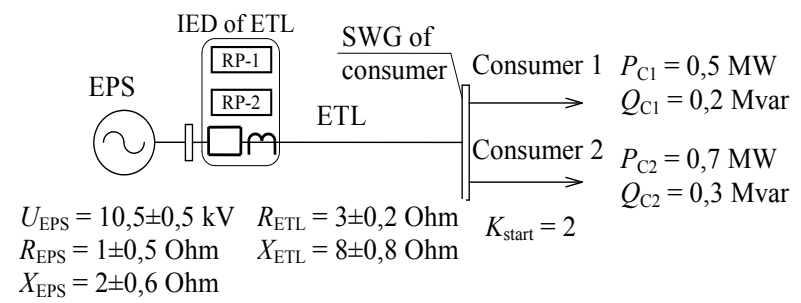

Fig. 2. Scheme for mode simulating

Example of the probability density functions for different modes, obtained based on mathematical modeling, in the observation space that includes only one monitored parameter (current) is shown in Fig. 3. Curves of probability density functions for various modes in multidimensional observation spaces can be drawn in a similar way and they depend on several parameters.

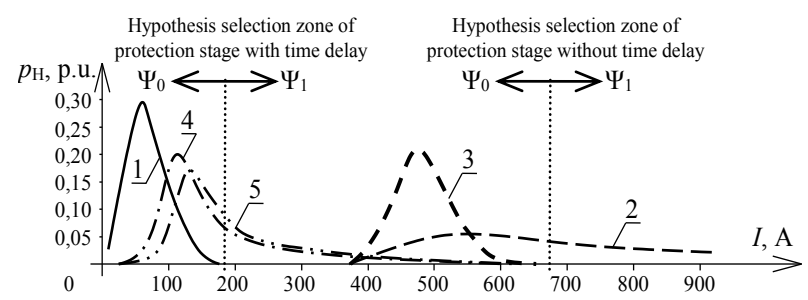

Fig. 3. Function of the facility mode conditional probability density for one-dimensional observation space: 1 - normal; 2 short-circuit in the supply line; 3 - short circuit fault in the SWG of consumer; 4 - short circuit fault in the consumer 1; 5 - short circuit fault in the consumer 2

Due to variability of characteristics of the factors affecting the protected facility condition, areas of its normal and emergency modes often overlap which does not allow protection to unmistakably assess its condition. Requirements of selectivity and inadmissibility of protection operation in normal mode allow setting the protection setpoints by comparing only the areas of different modes existence without taking into account numerical values of the conditional probability density. Example of such areas comparison for two-dimensional space (current, voltage) is shown in Fig. 4.
Probability of emergency condition recognition can be defined by integrating the function of conditional probability density for emergency conditions $p_{H_{1}}\left(\Phi \mid H_{1}\right)$ over the space of monitored parameters limited by the protection device setpoints $\left(\Psi_{1}\right)$. The values obtained for the protections monitored different sets of parameters are given in Table 1.

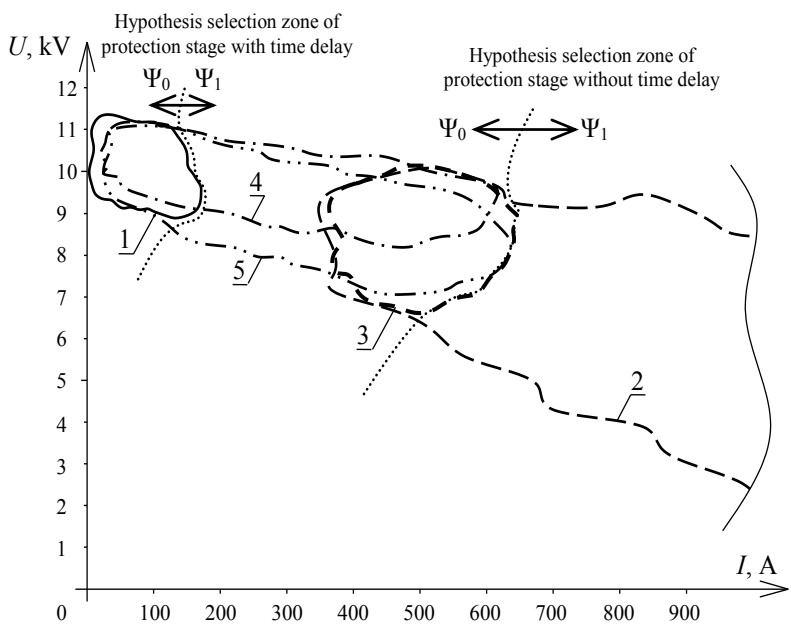

Fig. 4. Mode existence areas in two-dimensional observation space

Table 1. Comparison of probabilities of modes recognition by protection devices

\begin{tabular}{|l|c|c|c|c|}
\hline \multirow{2}{*}{ Recognition coefficients } & \multicolumn{4}{|c|}{ Monitored parameters } \\
\cline { 2 - 5 } & $I$ & $I, U$ & $P, Q$ & $I, U, \varphi$ \\
\hline $\begin{array}{l}\text { Probability of short circuit } \\
\text { fault recognition in a supply } \\
\text { electrical transmission line by } \\
\text { an instantaneous action stage }\end{array}$ & 0,597 & 0,806 & 0,795 & 0,898 \\
\hline $\begin{array}{l}\text { Probability of short circuit } \\
\text { fault recognition in a supply } \\
\text { electrical transmission line by } \\
\text { a stage with time delay }\end{array}$ & 1 & 1 & 1 & 1 \\
\hline $\begin{array}{l}\text { Probability of short circuit } \\
\text { fault recognition in the } \\
\text { consumer 1 by a stage with } \\
\text { time delay }\end{array}$ & 0,255 & 0,322 & 0,937 & 0,955 \\
\hline $\begin{array}{l}\text { Probability of short circuit } \\
\text { fault recognition in the } \\
\text { consumer 2 by a stage with } \\
\text { time delay }\end{array}$ & 0,367 & 0,461 & 0,950 & 0,958 \\
\hline $\begin{array}{l}\text { Total probability of short- } \\
\text { circuit fault recognition in } \\
\text { reserve zones by a stage with } \\
\text { time delay }\end{array}$ & 0,311 & 0,392 & 0,944 & 0,957 \\
\hline
\end{tabular}

\section{Hardware implementation of the proposed protection principles}

In the course of the research, a technical task for development of intelligent electronic device with a sufficient for the mentioned functions computing power was prepared. The device prototype presented by the research and production enterprise is shown in Fig. 5. The device feature consists in its ability to function at computing platform of any manufacturer that, depending 
on mathematical calculation complexity, allows using various productivity microprocessors of both domestic and foreign production without significant structural changes.

Integration into information networks and implementation of the concept for protection of micronetworks with distributed generation sources is carried out by means of fully-fledged use of IEC 61850 data transfer protocol.

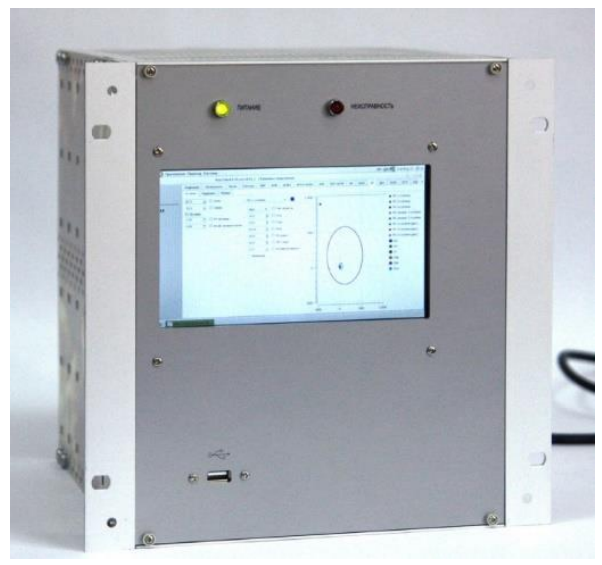

Fig. 5. View of the intelligent electronic device

\section{Software package for automatic calculation of the protection setpoints}

It is reasonable to carry out configuring and adapting of relay protection and automatic system elements parameters for current operating conditions of the micronetwork, including its topology, using technology of automatic calculation and matching of the relay protection. It implemented in a centralized device or a set of decentralized devices that receive information about the micro-network condition using IEC 61850 network communication standard.

Components of the software layout implementing the automatic calculation technology are shown in Figure 6.
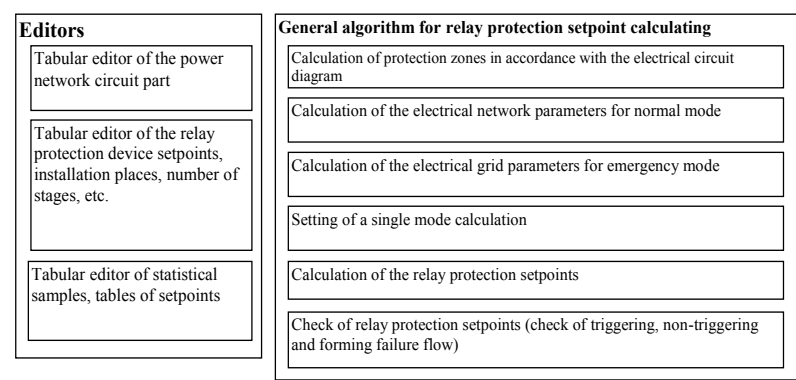

Fig. 6. Components of the software system for automatic calculation and matching triggering parameters of the network relay protection devices

\section{Conclusions}

1. The concept of relay protection system in micronetworks with distributed generation sources involving use of two-level relay protection scheme, elements of which use IEC 61850 protocol to exchange of information among themselves, is proposed.

2. Bayesian principle, which applies probabilities of protected element possible conditions, was used as a basis of the method for detection of protected element emergency modes.

3. Probabilities of faults detected by protection devices, which monitor different sets of the facility parameters, were compared.

4. An intelligent electronic device which implements the concept of micro-network protection involving a dynamically changing topology and generation, as well as a software package for automatic calculation of current protection setpoints on the basis of this concept, was developed.

This work was supported by the Ministry of Science and Higher Education of the Russian Federation (agreement No. 075-15-2019-1209 of May 31, 2019. Unique project identifier RFMEFI57717X0244).

\section{References}

1. O. A. Onisova, EEPIR J., 5, 88 (2018).

2. O. A. Onisova, Energetik J., 1, 17 (2015).

3. P. V. Ilyushin, RZA J, 2, 12 (2018).

4. TerzijaY. Proceedings of the IEEE., 1(99), 80 (2011).

5. A. L. Kulikov, M. V. Sharygin, El. St., 3, 37 (2018).

6. A. L. Kulikov, M. V. Sharygin, Izv. Ac. N. Energetika, 6, 38 (2018).

7. V. I Nagay, Izv. VUZ. Elektromechanika, 6(60), 84 (2017)

8. A. L. Kulikov, M. V. Sharygin, Elektrichestvo, 7, 20 (2017)

9. A. L. Kulikov, M. V. Sharygin, Elektrichestvo, 9, 24 (2017)

10. P. Anil Kumar, IJACEEE, 1(1), 19 (2013).

11. G. S. Nudelman, O. A. Onisova, EEPIR J., 4, 106 (2014). 